\title{
Interfertility and clonal size in the Armillariella mellea complex
}

\section{KARI KORHONEN}

\section{Introduction}

Armillariella mellea was originally described as Agaricus melleus in Flora Danica by Vahl (1790). Fries (1821) again described it on the basis of Swedish material and placed in the tribus Armillaria. Later Kummer (1871) and Quélet (1872) raised Armillaria to an independent genus. Karsten (1881) placed $A$. mellea in the genus Armillariella.

In the latest twenty years altogether about twenty taxonomical species belonging to the $A$. mellea complex (which includes those Armillariella species with a distinct ring) have been described in the world and about seven are claimed to occur in Europe (Singer 1956, 1975, 1977, Romagnesi 1970, 1973). To date, however, knowledge about the composition of this group and especially about the distribution and biological properties of the individual species is deficient.

Recently, genetical and cytogenetical studies have been directed at the $A$. mellea complex. As regards sexuality, ' $A$. mellea' has earlier been suspected as being homothallic (Raper 1966). This view was supported by the cytological peculiarity of this fungus: the hyphal tips are uninucleate. In addition, Kniep (1911) reported that in pure culture the four- spored basidia may arise directly from uninucleate cells of the vegetative mycelium, without fusion of nuclei. On the other hand, it is known that basidia in the basidiocarps of ' $A$. mellea' arise from clamped dikaryotic hyphae and show nuclear fusion before meiosis (Tommerup \& Broadbent 1975, Peabody et al. 1978).

In mating experiments with basidiomycetes the heterokaryotic mycelium produced in a compatible mating has usually been recognized by the presence of clamp connections. In a vegetative mycelium of ' $A$. mellea' there are no clamps. Hintikka (1973) found, however, that the single-spore isolates, with cottonous aerial mycelium, differ in their appearance from the flat crustose mycelium isolated from the flesh of the basidiocarps, from rhizomorphs, or from infected wood. In pairings between single-spore isolates the crustose mycelial type was formed in accordance with the tetrapolar (bifactorial) mating system.

Since the hyphal tips in both the monosporous mycelia and the crustose mycelia were uninucleate, Hintikka (1973) suggested that the nuclei in the latter mycelial type are diploid. While attempting to solve the diploidization mechanism, a transient dikaryotic 
phase with clamp connections was found in compatible matings (Korhonen \& Hintikka 1974). Similar unstable dikaryotic hyphae could be isolated from the gills of the basidiocarps. In the dikaryotic tip cells a somatic diploidization mechanism was found: the nuclei fused and the cell divided into two uninucleate cells. The monokaryotic mycelium originating from both the cells thus formed, represented the crustose mycelial type.

This observation raised the question about the life cycle: if the vegetative mycelium is diploid where do the clamped dikaryotic hyphae giving rise to basidia originate from? Tommerup and Broadbent (1975) found, by studying the size of the nuclei, that the dikaryotic hyphae arise via haploidization in young basidial primordia. The haploidization mechanism remained unknown.

Recently Ullrich and Anderson (1978) have confirmed the bifactorial mating system of ' $A$. mellea'. They also present genetic evidence for diploidization in compatible matings. The DNAmeasurements made by Peabody et al. (1978) also suggest a basically diploid life cycle with heteroploidy in some stages of the life cycle.

Species identificaton of wood rotting fungi on the basis of their pure cultures has proved valuable in forest pathological research. Many authors have referred to the differences in the external appearance of different 'A. mellea' isolates. Some morphological characteristics seem in actual fact to be typical of certain species (Jacques-Félix 1977) but generally speaking it is not known to what extent the cultural characteristics can be used as a means of species determination in the $A$. mellea complex. Determining the mating system made it possible to examine whether the Buller phenomenon could be used as an aid in identification of Armillariella pure cultures (Miles \& Raper 1956, Osborne 1970, Bruehl et al. 1975).

This study was started in the hope of obtaining a more precise picture of the relationships which exist between different members of the $A$. mellea complex. The following aspects have been investigated: (1) interfertility within the complex, (2) mating systems of different species, (3) species identification of diploid pure cultures with the aid of the Buller phenomenon, (4) biological properties of different species, and (5) clonal study methods and naturally occurring clones of Armillariella in Finland.

\section{Material and methods}

The standard nutrient medium contained $1.5 \%$ Difco malt extract and $1.5 \%$ agar. The pure cultures were incubated in the dark at room temperature $\left(20-23^{\circ} \mathrm{C}\right)$ and stored on malt extract agar slants in test tubes at $4^{\circ} \mathrm{C}$, transfers being made once a year. For pairing, two cultures were inoculated $0-2 \mathrm{~mm}$ apart from each other. Macromorphological mating interactions were examined after 3 weeks incubation. The cytological study methods were those of Korhonen and Hintikka (1974).

For interfertility studies, samples of fresh basidiocarps were collected from about 70 different localities in southern and Central Finland. In addition, some samples were collected in France. The collection localities in Finland were chosen rather arbitrarily except for some Armillariella infected pine plantations. Most of the material was collected near Helsinki. In most cases the area within which the basidiocarps were sought was about $200 \mathrm{~m}$ in diameter. For clonal studies, several basidiocarps were collected from the same locality and their approximate position in the field was recorded.

About 10-12 single-spore cultures were isolated from each basidiocarp sample. In addition, a diploid culture was isolated from the flesh of the basidiocarp. The basidiocarp samples were dried at $40-50^{\circ} \mathrm{C}$ and stored for morphological studies. The term 'stock' in this study refers to one sample (a naturally occurring mycelium with possible basidiocarps) and includes all single-spore cultures isolated from it.

Different mating types (Raper 1966) among single-spore isolates of each stock were worked out, usually by pairing 8 isolates in all combinations. Whenever necessary, the remaining isolates in each stock were tested with the different types found in the first pairings.

In order to study different $\mathrm{A}$ and $\mathrm{B}$ incompatibility factors within a population, and to test for the existence of genetical isolation mechanisms between stocks, all four mating types from one stock were paired with two compatible mating types from another stock. In addition, interfertility experiments were carried out in which each stock was represented by only one or two monosporous isolates. The study material was divided into intersterile groups on the basis of experiments carried out with singlespore mycelia.

The existence of the Buller phenomenon in pairings between diploid and haploid mycelia was then investigated. The Buller phenomenon was used in identification of unknown diploid pure cultures of the $A$. mellea complex as follows: Four single-spore isolates were chosen from each intersterility group. These isolates showed a clear morphological change in compatible matings and were used as testers in diploid-haploid pairings. An unknown diploid isolate was paired with all the testers and the change of the tester into the crustose mycelial type was recorded after $3-5$ weeks incubation.

The study material comprises single-spore cultures from about 400 Finnish and 26 French basidiocarp samples as well as from one Norwegian, one German, and one Byelorussian sample. In addition, about 150 stocks represented by a diploid culture only were examined with the aid of the Buller phenomenon. Among them, about 120 originate from Finland, 10 from Norway, 10 from New Zealand, 5 from France, 4 from Poland, 3 from England, and one from Tanzania.

Single-spore isolates from two distinctly described species within the $A$. mellea complex were included in the mating experiments: $A$. mellea (Vahl ex Fr.) Karst. in the sense of Romagnesi (1973) and $A$. bulbosa (Barla) Romagn. The former species was represented by 19 stocks and the latter one by 7 stocks with single-spore mycelia. This material was isolated by the author in France. Dr. Henri Romagnesi, 


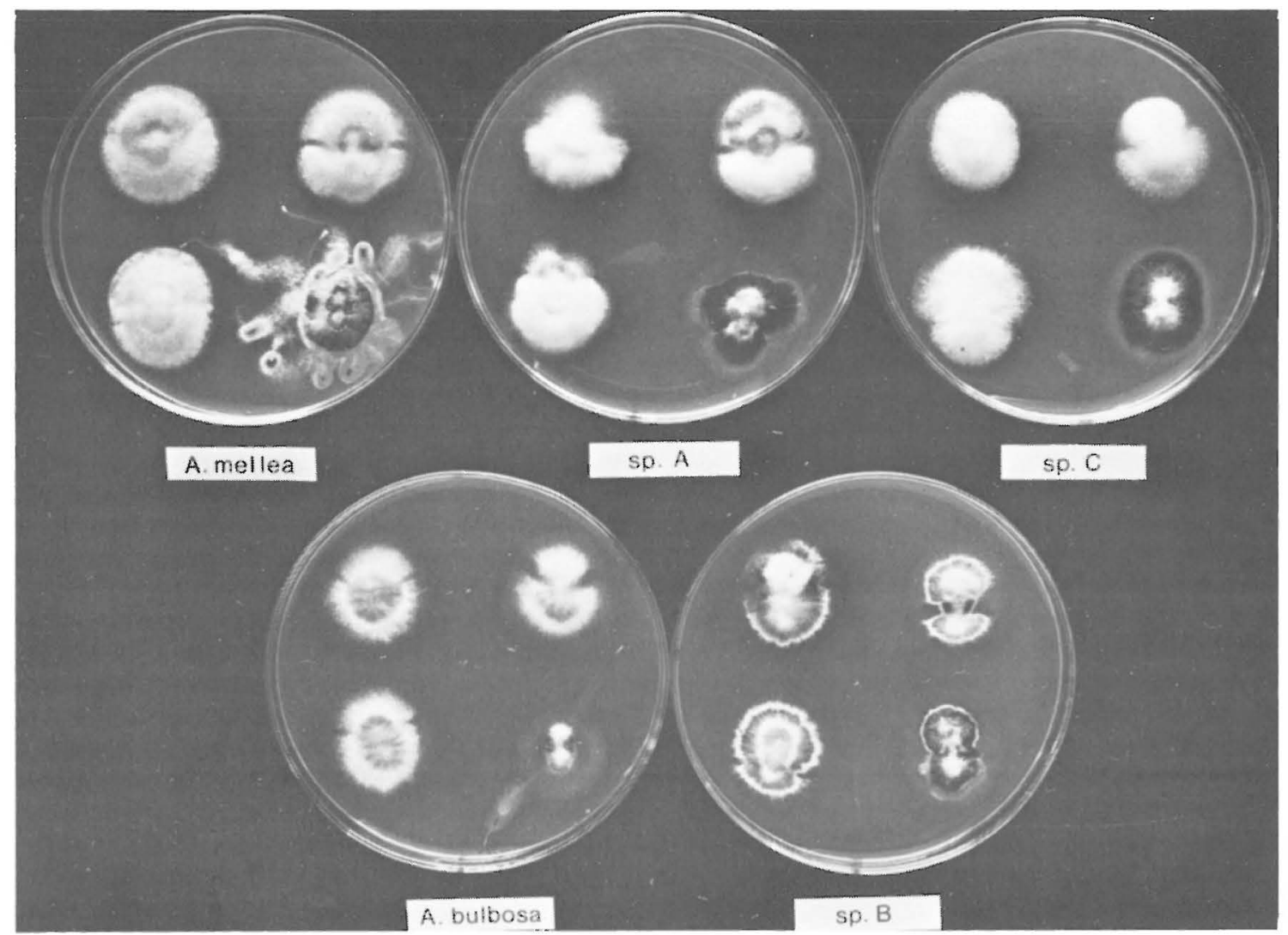

Fig. 1. Appearance of different incompatibility factor combinations in matings of the studied species. In each dish the upper left mating is incompatible, upper right hemicompatible common-A, lower left hemicompatible common-B, and lower right compatible. The lower mycelium in each of the four matings shown in the dish is the same. Age 20 days. Note a broad barrage zone in most hemicompatible common-A matings.

Muséum National d'Histoire Naturelle, Paris, and Dr. Micheline Jaques-Félix, Institut National Agronomique, Paris, have kindly identified the basidiocarp samples. In mating tests diploid cultures of the species $A$. novaezelandiae Stevenson and A. limonea Stevenson, kindly provided by Dr. Charles G. Shaw III, Forest Research Institute, Rotorua, New Zealand, were also included.

\section{Results and discussion}

\section{Intersterile groups}

The study material consisting of single-spore isolates was divided into five intersterile groups. Excluding the effect of identical incompatibility factors, each group was completely intrafertile. No signs of hybridization between different groups were detected in about 4000 inter-group matings.

The species $A$. mellea (Vahl ex Fr.) Karst. sensu stricto formed one intersterility group and $A$. bulbosa (Barla) Romagn. another one. The species concept of
Romagnesi (1973) was thus genetically confirmed. The morphology of the basidiocarps and the nomenclature of the remaining three intersterile biological species have not yet been thoroughly studied. In this paper they are referred to as 'species $\mathrm{A}, \mathrm{B}$, and $\mathrm{C}^{\prime}$.

\section{Mating systems and interactions}

The studied material is consistent with the finding of Hintikka (1973) that the cottonous mycelial morphology of monosporous pure cultures of ' $A$. mellea' is different from that of pure cultures isolated from the flesh of the basidiocarps or from vegetative mycelia in vivo. The latter crustose mycelial type is the result of compatible mating between monosporous mycelia.

All the five species studied exhibit a heterothallic bifactorial mating system (Raper 1966). No attempt 
was made to determine the approximate number of different A and B incompatibility factors, but on the basis of the mating experiments performed it can be concluded that the number of different $\mathrm{A}$ and $\mathrm{B}$ factors in each species is more than fifteen.

The external mating interactions were about the same in all the species studied and are presented below and in Fig. 1. However, according to preliminary observations, $A$. mellea s. str. seems to exhibit some marked cytological differences.

1) Incompatible mating $(\mathrm{A}=\mathrm{B}=)$. No macro- or microscopical mating interactions were found (hyphal fusions were not examined). The mycelia grow densely side by side.

2) Hemicompatible common-B mating $(A \neq B=)$. Usually similar to an incompatible mating.

3) Hemicompatible common- $\mathrm{A}$ mating $(\mathrm{A}=\mathrm{B} \neq)$. In a typical case there is a 'barrage zone' between the confronting mycelia where the aerial mycelium is sparse or lacking. When examined under the microscope, partially disintegrated septa can be found in some of the submerged hyphae in this zone. This points to nuclear migration and indicates that the B incompatibility factors of the mating mycelia are different (Raper 1966).

4) Compatible mating $(A \neq B \neq)$. The border between the mating mycelia disappears and the morphology of the mycelia changes into the crustose type. In some hyphae there are disintegrated septa and migrating nuclei. In the species $\mathrm{A}, \mathrm{B}, \mathrm{C}$, and $A$. bulbosa there is a transient phase of clamped dikaryotic hyphae. In all these species a similar monokaryotization mechanism via somatic diploidization in dikaryotic tip cells was observed, as presented earlier (Korhonen \& Hintikka 1974). Clamped hyphae have not so far been found in $A$. mellea s. str. and the diploidization mechanism is unclear. [The paper by Korhonen and Hintikka (1974) does not deal with $A$. mellea s. str. The study material included pure cultures of species A and B. The study object in Hintikka's (1973) paper belongs to species B.]

There are minor differences in mating interactions between different species and stocks. For instance, in a hemicompatible common-A mating there is often a zone of crustose mycelium on the barrage line (species B in Fig. 1). Sometimes it may even spread into one or both mating partners and causes difficulties in distinguishing between this combination and a compatible one.

It seems to be typical of $A$. mellea s. str. that its diploid mycelium often does not become crustose but instead forms a short dense aerial mycelium and many rhizomorphs (Fig. 4d). However, this mycelial type is so different from the higher and less dense aerial mycelium of single-spore isolates that the morphological change in a compatible mating is normally clear.

On the other hand, the study material includes stocks $(5-10 \%)$ from all those species, where it is difficult to find macromorphological mating interactions. These stocks were not thoroughly investigated. The reason for unclear mating reactions may be, for example, lack of compatible mating types among single-spore cultures, unilateral maters (Miles \& Raper 1956), or degeneration. The degeneration of haploid isolates is a problem in storing the cultures but it cannot be the only reason for those unclear reactions. When single-spore isolates have been stored for 3-5 years on malt extract agar medium at $4^{\circ} \mathrm{C}$ a large proportion of them show decreased mating ability. Together with degeneration the ability to form aerial mycelium usually weakens.

Unstable dikaryotic hyphae with clamp connections were isolated and cultivated from $1-2$ week old compatible matings of species A, B, C, and $A$. bulbosa in a similar way as the dikaryotic hyphae were earlier isolated from the basidiocarps (Korhonen \& Hintikka 1974).

Spreading of diploid hyphae into the mating mycelia is a slow phenomenon in Armillariella as

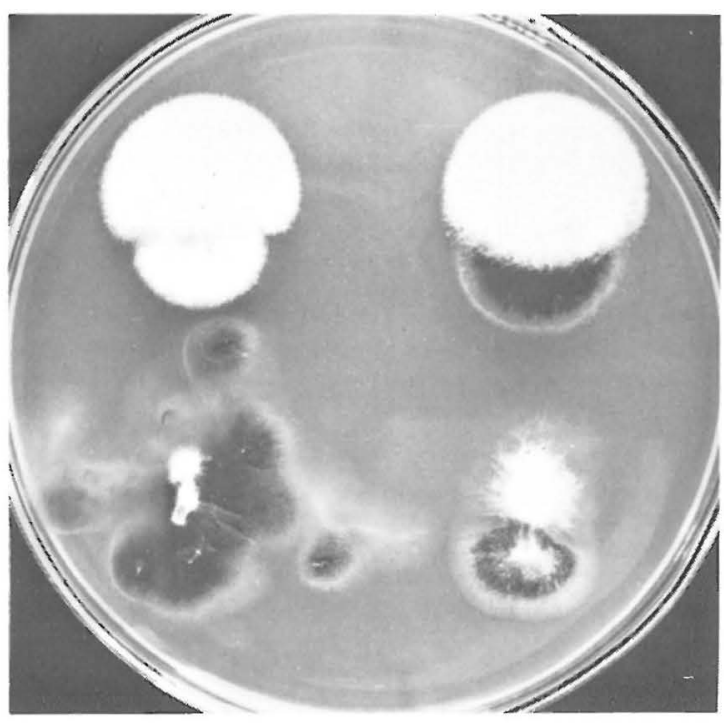

Fig. 2. Typical reactions of a haploid tester strain in mating test. The upper mycelium in each pairing is the same tester belonging to species $\mathrm{A}$. The lower mycelium: upper left a haploid mycelium of species $C$, upper right a diploid mycelium of species $\mathrm{C}$, lower left a compatible haploid mycelium of species $\mathrm{A}$, and lower right a diploid mycelium of species A. Age 20 days. 
compared with heterokaryotization in some other hymenomycetes (Ross 1976). In species A single haploid hyphal tips can often still be isolated from two or three-week old compatible matings, especially in intrastock matings. This suggests that the diploidization process is somewhat faster in interstock matings, thus favouring outbreeding. Attempts to isolate haploid or dikaryotic hyphal fragments from old diploid cultures were unsuccessful.

\section{Species identification of diploid cultures with the aid of the Buller phenomenon}

The Buller phenomenon in its original sense is concerned with fungi in which the nuclei are haploid. The nuclei in a vegetative mycelium of Armillariella are, however, probably diploid. In spite of diploidy it was found that a phenomenon corresponding to the Buller phenomenon is also operating between the diploid and haploid mycelia of Armillariella. When a cottonous haploid mycelium is paired with a crustose diploid mycelium, the appearance of the haploid mycelium usually changes to the crustose mycelial type. This change is often slow as compared with the change taking place in matings between two compatible haploids (Fig. 2), but it can be used as an aid in identifying the diploid pure cultures. A haploid mycelium preserves its original appearance when it is paired with a mycelium belonging to different species in the $A$. mellea complex.

In order to study the usefulness of the Buller phenomenon in species identification, about 250 diploid pure cultures of Armillariella were tested by pairing them with four haploid testers from each of

Table 1. The number of stock cultures of the European Armillariella species and their geographical distribution in the study material

A. mellea s. str. 25 France, near Paris 23 (Eure, Oise, Seine et Oise, Seine et Marne), Pas-de-Calais $1^{1)}$, Périgord $1^{1)}$,

A. bulbosa 11 France, near Paris 8; England, species A Thetford $3^{1)}$

species A $\quad 377 \quad$ Finland 371; Norway, near Oslo $4^{1)}$, Troms 1); Byelorussia, Minsk $1^{2)}$.

species B 93 Finland 86; Norway, near Oslo 6, species C $\quad 47 \quad \begin{aligned} & \text { Germany, Munich 1. } \\ & \text { South Finland 43; Poland 4), }\end{aligned}$

1) diploid cultures only, identified with the Buller phenomenon

2) with somewhat unclear mating reactions the species A, B, C, $A$. mellea s. str., and $A$. bulbosa. In a normal case the diploid pure culture belonging to any of the tester species could be unambiguously identified. In a few cases, however, the results were more difficult to interprete, especially when the tester and the diploid isolate originated from areas far away from each other. The isolates of $A$. novaezelandiae, $A$. limonea, as well as the ' $A$. mellea' isolate from Tanzania did not react with these testers.

The change in the haploid mycelium which takes place in diploid-haploid pairing presumably requires the presence of some haploid nuclei in the diploid mycelium. However, preliminary attempts to isolate haploid mycelial fragments originating from the diploid mycelium in diploid-haploid pairings were unsuccessful. Koltin and Raper (1968) found that nuclei in an exceptional diploid strain of Schizophyllum commune were unstable and haploidized when the mycelium was paired with a haploid homokaryon.

When diploid or haploid mycelia belonging to different biological species of Armillariella are confronted they almost invariably produce brown pigment along the demarcation line (Fig. 5). It can be seen best by examining a petri dish culture through the bottom glass. This reaction was found between all the species included in mating tests. It can be used as an additional aid in species identification. Ullrich and Anderson (1978) have noted a similar phenomenon in the American Armillaria material. Somewhat similar reactions, although not solely restricted to interspecies confrontations, have been observed in other fungi, too (Edwards \& Kennedy 1973).

Table 2. The number of studied Finnish Armillariella stocks isolated from different habitats.

Wood with advanced rot (old stumps, etc.)

Picea abies

Pinus sylvestris

other conifers

Betula sp.

other deciduous trees

Species

Living trees or recently dead ones, the

cause of death possibly Armillariella

Picea abies, butt rot

Pinus sylvestris, trees
" $\quad$ saplings
other conifers (Larix sibirica)
Betula sp.
Other deciduous trees
known
tal

$\begin{array}{rrr}\text { A } & \text { B } & \text { C } \\ 119 & 9 & 1 \\ 9 & 2 & 1 \\ 3 & 2 & - \\ 83 & 17 & 2 \\ 34 & 10 & 1\end{array}$

$\begin{array}{rrr}51 & 10 & - \\ 6 & 2 & - \\ 2 & - & - \\ 5 & 3 & 37 \\ 4 & - & - \\ 13 & - & - \\ 6 & 6 & 1 \\ 36 & 25 & - \\ 371 & 86 & 43\end{array}$

$$
\begin{aligned}
& \text { C } \\
& 1 \\
& - \\
& 2
\end{aligned}
$$




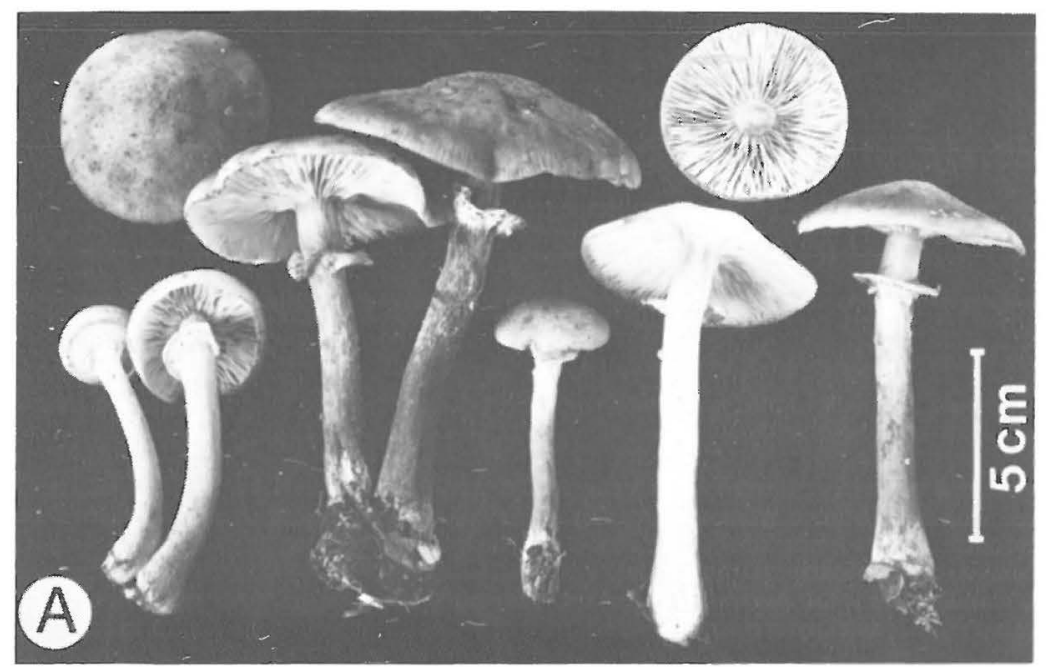

Fig. 3. The basidiocarps of the Armillariella species A, B, and C.
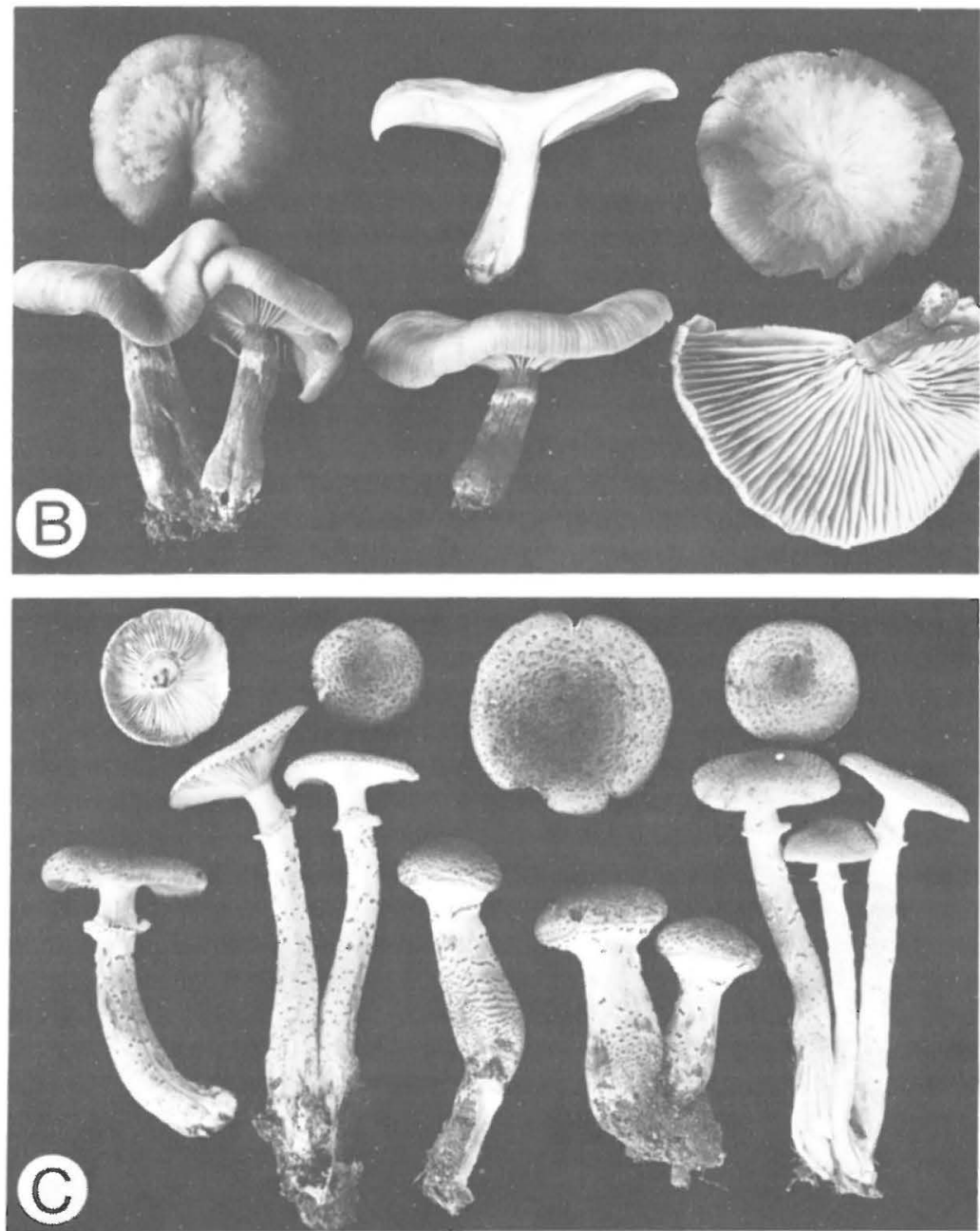

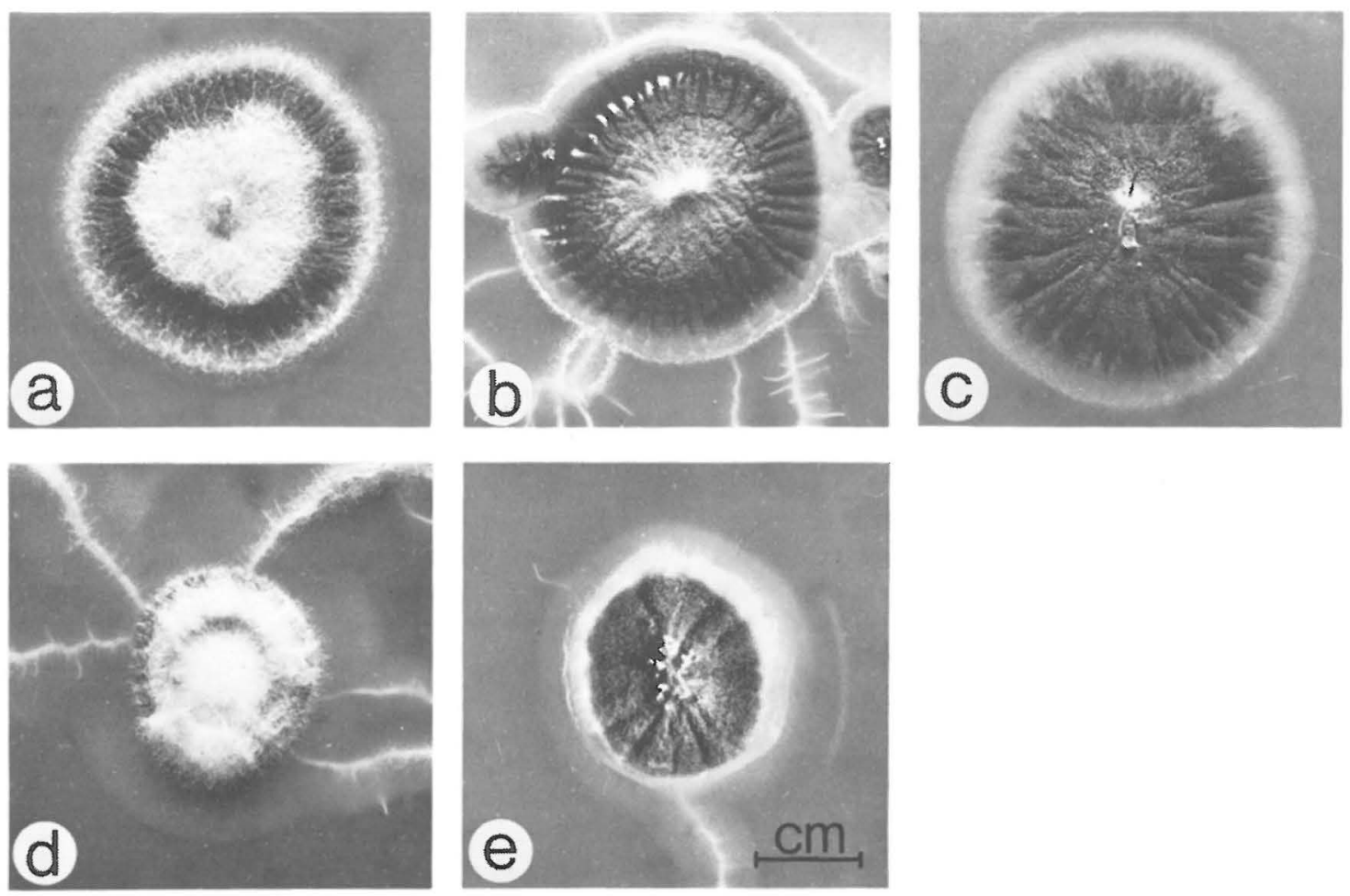

Fig. 4. Diploid pure cultures of a) species A, b) species B, c) species C, d) A. mellea s. str., e) A. bulbosa. Age of cultures 20 days.

\section{Notes on the properties of different species}

The division of the European study material into different species and geographical areas is presented in Table 1, and the distribution of the Finnish material into different habitats in Table 2. Some notes on the morphological and ecological characteristics of the Finnish species are presented below.

The basidiocarps of species A (Fig. 3) resemble $A$. mellea s. str. However, there seems to be at least one clear and interesting difference between these species: the basidium of $A$. mellea s. str. does not have a basal clamp (Romagnesi 1973) whereas the clamp seems to occur regularly on the basidium of species A. The cap of this species bears brown scales, the stipe is usually long and of uniform thickness, the ring is thick. Rhizomorphs are chestnut brown, usually flat. The diploid pure culture (Fig. 4a) is a dull crustose mycelium, sometimes submerged.

Species $\mathbf{A}$ is common in southern and Central Finland. The most northern record in the study material is from Sodankylä. The basidiocarps appear, often in great quantities, in August or in September, sometimes also later in the fall. This species usually grows as a saprophyte in the wood of many tree species. It has also been found on many living trees, probably as a secondary parasite. It seems to be the commonest Armillariella species in the butt rot of Picea abies (L.) Karst., although its frequency in southern Finland is generally much lower than that of Heterobasidion annosum (Fr.) Bref. (Kallio \& Tamminen 1974).

Species B (Fig. 3) resembles $A$. bulbosa. The scales on its cap are small and their colour is usually dark grey. The base of the stipe is typically swollen. The ring is thin, arachnoid. The rhizomorphs are dark brown, thin, cylindrical. The basidia are clamped. The diploid pure culture (Fig. 4b) is characteristically wrinkled, but often grows only submerged and then stains the agar medium deep brown.

This species seems to be fairly common in southern and Central Finland. The most northern record is from Rovaniemi. The basidiocarps usually appear later than those of species $\mathrm{A}$ and in some autumns they are difficult to find. Often they grow on very old stumps or on the ground apparently far away from the wood substrate. Ecologically this species 


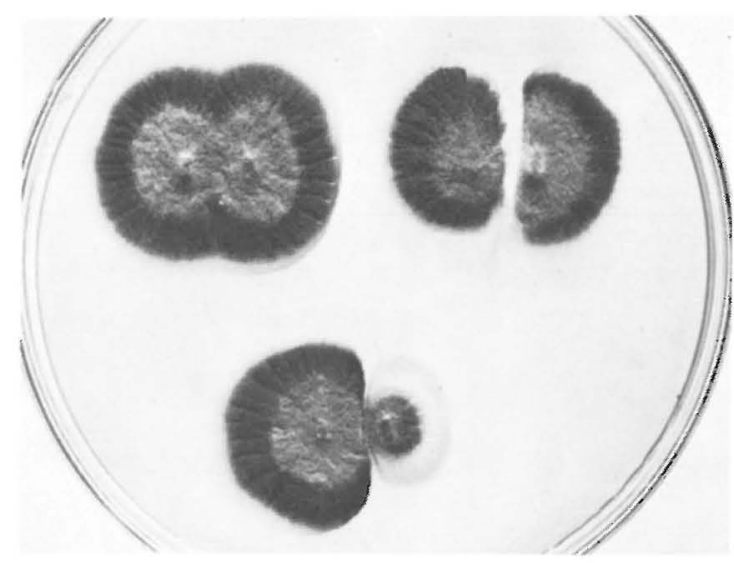

Fig. 5. Confrontations of diploid mycelia. Upper left two genetically identical mycelia of species A showing complete intermingling. Upper right genetically different mycelia of species A showing a demarcation line without pigment. Bottom, species $\mathrm{A}$ and species $\mathrm{C}$ confronted, showing a demarcation line with brown pigment. Age 20 days. Photographed in transmitted light.

resembles species $\mathrm{A}$, and sometimes they can be found growing in the same stump. Species B is also found to some extent in the butt rot of Norway spruce. In addition, it has been found growing on some living park trees (Acer, Syringa).

Species C (Fig. 3) is probably $A$. ostoyae Romagnesi (1970) although this has not so far been confirmed by mating experiments. It resembles species $\mathrm{A}$, but has large brown scales on the cap and stem. The basidia are clamped. The diploid pure culture (Fig. 4c) is a somewhat glossy crustose mycelium or submerged, staining lightly.

In Finland species $\mathrm{C}$ was found only in the southern part of the country. One sample was collected from living Alnus incana (L.) Moench in Helsinki, all the others from Pinus sylvestris L. plantations on the Salpausselkä chain of eskars (Tenhola, Lohja, Nurmijärvi, Hausjärvi and Luumäki). Apparently using pine or birch stumps as a food base, species $\mathrm{C}$ attacks pine saplings causing damage in the plantations (Hintikka 1974). The Polish samples of this species have also been isolated from Scots pine, and $A$. ostoyae is a pathogen of Pinus pinaster Ait. in Les Landes, France (JacquesFélix 1977).

The basidiocarps of species $\mathrm{C}$ appear in Finland fairly late in the fall, in September - November. Rykowski (1974 and pers. comm.) has succeeded in making this species fruit in pure culture, and it has produced somewhat deformed basidiocarps in our laboratory, too. Jacques-Félix (1977) reports that $A$. ostoyae fruits in vitro.

\section{Clonal studies}

Naturally occurring clones of Armillaria have been recently studied in the U.S.A. Adams (1974) and Shaw and Roth (1976) found in Pinus ponderosa stands remarkably large clones with a diameter of up to more than one kilometer and an estimated age of at least 460 years. The clones studied by Ullrich and Anderson (1978) were much smaller, the largest one having a diameter of $50 \mathrm{~m}$.

When collecting material for this study several samples were usually taken from the same locality infected by Armillariella. Some of their genetical properties were compared in order to get some idea about the size of the clones and about their infection power towards different tree species in Finland. These studies were performed at about 40 localities in southern and Central Finland. At least three basidiocarp samples were collected from each locality. At a few localities the clones were thoroughly investigated and, in addition to basidiocarps, diploid isolates were collected from infected wood. The clonal study material for species A comprises 210 basidiocarp samples from 30 localities, for species B 44 samples from 9 localities, and for species C 21 samples from 4 localities.

Two methods were used in identifying the clones: 1) A and B incompatibility factors were used as genetic markers (Martin \& Gilbertson 1978, Ullrich \& Anderson 1978). All basidiocarps belonging to the same clone should contain identical incompatibility factors. However, with this method it is not possible to distinguish inbred sibling clones from each other or from their parent clone.

2) The diploid isolates were confronted with each other. Genetically identical mycelia intermingle completely, but genetically different ones form a permanent demarcation line. The brown pigment is lacking in intra-species confrontations (Fig. 5). This method has been applied to 'A. mellea' by Adams (1974) and Shaw and Roth (1976), but they have recorded the dark line of demarcation on different nutrient medium.

These two methods gave almost identical results in the study material which consisted of about 110 clones. If the samples collected from the same locality contained identical incompatibility factors, then their diploid pure cultures also intermingled completely. If the samples differed even in one factor their diploid cultures were separated by a permanent demarcation line.

However, three exceptional cases were found. One clone of species A (clone 2 in Fig. 7), of which there were many basidiocarp samples (22), was homogenous as judged on the basis of demarcation line formation. On one side of the clone four mating types were equally represented among 87 single-spore isolates collected from 8 basidiocarp samples, but on 


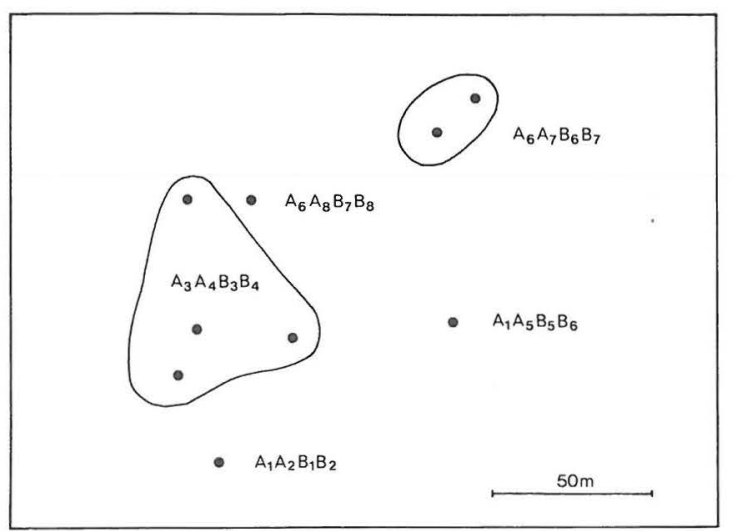

Fig. 6. Clones of Armillariella species A from an infected area in a spruce forest mixed with birch at Seutula, about 25 $\mathrm{km}$ north of Helsinki. The samples were collected from spruce and birch stumps. The incompatibility factors of each clone are marked.

the other side two mating types were almost completely lacking from the 113 single-spore isolates from 14 basidiocarp samples. The missing types had a common B factor. This factor was found only in four single-spore isolates whereas the expected value is $113 / 2$. The rare B factor seemed to be different from the normally distributed $\mathrm{B}$ factors of the clone, but the reactions were somewhat unclear. The reason for this discrepancy could be a mutation in one B factor or in the same chromosome near it, combined with a high degree of lethality.

In two other exceptional cases the samples of species A collected near each other contained identical incompatibility factors, but in confrontations the diploid isolates were separated by a permanent demarcation line. One explanation for this is that the samples represented a parent clone and an inbred $F_{1}$ clone, or two $F_{1}$ clones. Another explanation could be a mutation in a part of the clone, becoming visible in confrontation test.

To test the previous hypothesis a preliminary experiment was arranged in which members of inbred diploid progeny were confronted with each other and with their parent mycelium. The $F_{1}$ diploids were isolated as single hyphal tips from compatible matings between monosporous mycelia belonging to the same stock. Altogether 47 inbred diploids were isolated from four parent stocks of species A. Among 132 different combinations of sibling diploids from one parent stock a permanent demarcation line was formed in 68 cases and the mycelia intermingled completely in 64 cases. The corresponding values for

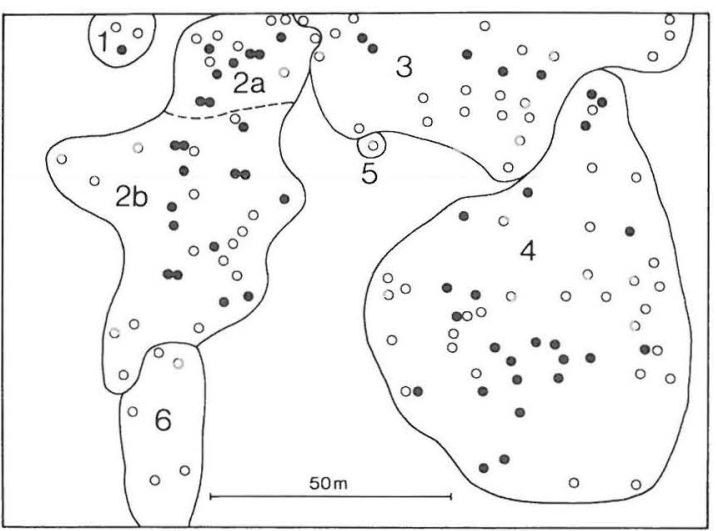

Fig. 7. Clones of Armillariella species A at a heavily infected locality in a mixed forest consisting of spruce, birch, and pine, Pohjois-Haaga, Helsinki. The forest has been greatly affected by man's activities and contains many stumps. Solid circle: incompatibility factors determined and diploid isolate studied in confrontations. Open circle: only diploid isolate studied. Incompatibility factors of the clones: (I) $\mathrm{A}_{1} \mathrm{~A}_{2} \mathrm{~B}_{1} \mathrm{~B}_{2}$, (2a) $\mathrm{A}_{3} \mathrm{~A}_{4} \mathrm{~B}_{3} \mathrm{~B}_{4}$, (2b) probably $\mathrm{A}_{3} \mathrm{~A}_{4} \mathrm{~B}_{3} \mathrm{~B}_{5}$ (see text), (3) $\mathrm{A}_{3} \mathrm{~A}_{5} \mathrm{~B}_{6} \mathrm{~B}_{7}$, (4) $\mathrm{A}_{6} \mathrm{~A}_{7} \mathrm{~B}_{8} \mathrm{~B}_{9}$. In spite of identical numbering with Fig. 6 the factors are not the same.

parent stock two were 32 and 34 , for parent stock three 8 and 7, and for parent stock four 42 and 19 (with somewhat unclear reactions). The parent diploid also forms a demarcation line with some inbred $\mathrm{F}_{1}$ diploids but fuses with the others.

In species $\mathrm{A}$, the demarcation line is thus formed in about half of the combinations between inbred sibling diploids. A simple genetical mechanism seems to determine the formation of the demarcation line in these confrontations but in this preliminary experiment it was not thoroughly studied. In confrontations between sibcomposed dikaryons of Fomes (Fomitopsis) cajanderi with each other or with their parent dikaryon a demarcation line was formed in 59-83 \% of cases (Adams \& Roth 1967).

Neither of these two clonal study methods can thus definitely distinguish closely related clones of species A from each other. However, it seems that a fairly satisfactory picture of the size of clones can be obtained with either one of these methods. On the basis of the studied material it seems that the probability of the formation of inbred clones in nature is not high in spite of large amounts of spores being deposited near basidiocarps. There are apparently several reasons for this: (1) The aerial distribution of fungal spores seems to be effective (Kallio 1970). (2) The infection power of Armillariella spores is apparently weak (Rishbeth 1970), thus 


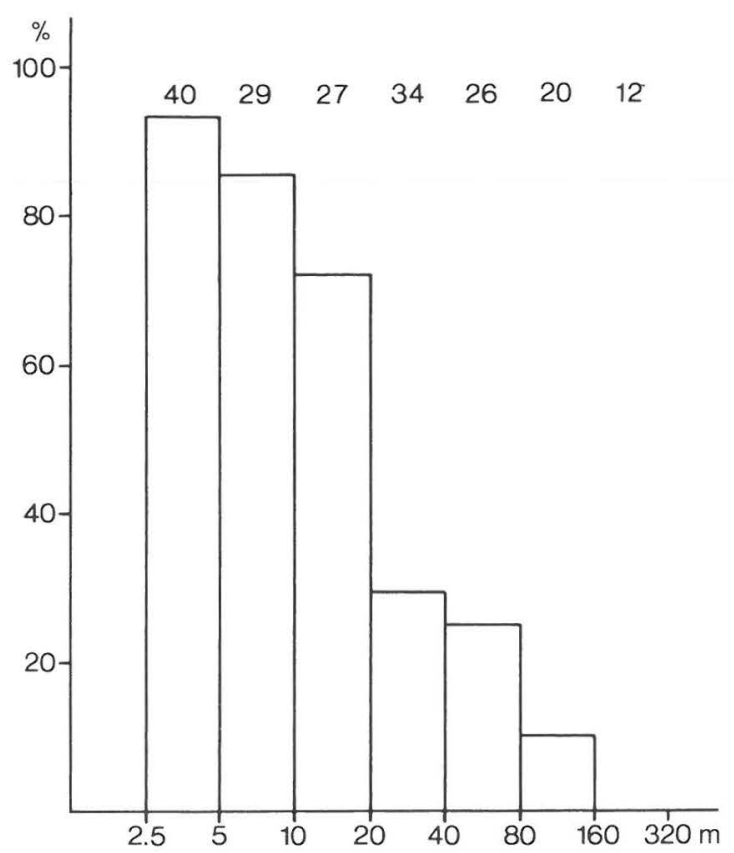

Fig. 8. Relation of distance between two samples to clone in species A material collected from southern and Central Finland. The distance is measured from the previous sample during collection. $\mathrm{X}$-axis: distance between two samples, in classes. Y-axis: cases where the samples belong to the same clone, per cent in each distance class. The number of recordings in each distance class is shown.

suppressing the formation of new clones. (3) The bifactorial mating system favors outbreeding. (4) As presented earlier, even the rate of the diploidization process of Armillariella (species A) seems to favour outbreeding.

The clonal study material for species A consisting of 210 basidiocarp samples from 30 localities was divided into 80 clones. There were, on the average, 2.7 clones per collection locality (with a diameter of about $200 \mathrm{~m}$ ) infected with species A (Fig. 6). The clones in a few localities were studied thoroughly (Fig. 7). The material does not permit very precise conclusions to be drawn about the average size of the clones but a rough estimate can be obtained from the distances recorded between collected samples (Fig. 8). The clonal study materials for species B and C are too small to make general conclusions, but they suggest that the average size of clones for these species is about similar to that for species A.

The largest clones found were about $120-150 \mathrm{~m}$ in diameter. The age of such clones in Finnish conditions is perhaps near to 100 years (Rishbeth 1968, Hintikka 1974, Shaw \& Roth 1976). However, the possibility that these large clones consist of two or more closely related clones cannot be ruled out. Generally, the size of Armillariella clones in Finland is smaller, a diameter of $10-50 \mathrm{~m}$ is usual.

No distinct specialization by different clones of the same Armillariella species to different tree species was found. As a rule, a single clone seems to be able to attack the same host species as the species in general.

The small size of collection localities in this study causes some uncertainness as regards large clones. In any case, most clones of Armillariella in Finland are relatively small, approximately the same size as those studied by Ullrich and Anderson (1978) in New England and much smaller than those found by Adams (1974) and Shaw and Roth (1976) in Pinus ponderosa stands of Oregon and Washington. In Finland the Armillariella species seem to be largely favoured by man's activities. This may be one reason why most clones are not old. In contrast, Armillaria is considered to have been present in Pinus ponderosa stands prior to any forest modification carried out by man. A question perhaps worth considering in connection with large and old clones is for how long a time the originally identical mycelium growing in different directions remains identical. With time, different mutations may accumulate in different parts of the clone and become visible in the confrontation test.

\section{Conclusions}

The results of the interfertility studies presented here are consistent with the view of taxonomists that ' $A$. mellea', as it is usually understood in the forest pathological literature, is a species complex. The studies by Ullrich and Anderson (1978) have shown that 'A. mellea' in North America also consists of several intersterile biological species.

The distinction of different species on the basis of morphology seems to be difficult in the A. mellea complex. In Europe the present state of taxonomy of this group is partially confusing. It is also possible that the present species concept is not yet final.

From the point of view of forest pathology it would be useful to elucidate this complex, if possible. The mating experiments seem to offer a good method for studying it. The mating tests can perhaps also be used in routine pure culture identification of the Armillariella species. It might be worth investigating if the dark pigment line formed in confrontations between diploid mycelia would alone offer a reliable basis for species identification. 
The question about life cycle in the $A$. mellea complex is especially interesting. The available evidence suggests that some species in this complex reqularly show two diploidizations and two haploidizations in their life cycle. Such species are apparently those ones which have clamped basidia originating from dikaryotic hyphae: species A, B, C, and $A$. bulbosa in this study. In addition, this complex seems to include species with clampless basidia. Such a species is $A$. mellea s. str. The behaviour of nuclei in these clampless basidia is so far unknown. It would be natural to suppose that the basidia arise directly from uninucleate diploid cells, in a similar way as did apparently the 'Mycelbasidien' observed by Kniep (1911). If this is the situation, the life cycle in this genus could also proceed without haploidization in young basidial primordia. Ullrich and Anderson (1978) suggest this kind of a diploid life cycle for the American Armillaria.

However, the recent DNA measurements carried out by Peabody et al. (1978) suggest that the nuclear cycle in the $A$. mellea complex may be even more complicated. Their results, based on one 'A. mellea' isolate, indicate that the basic life cycle is indeed diploid. The lamellar tramal tissue, however, seems to be tetraploid and a degree of heterogeneity in ploidy levels may occur in some stages of the life cycle at least.

The accumulated genetic evidence suggests that mitotic recombination is a rather common phenomenon among higher fungi. It requires the formation of transient diploid nuclei in the heterokaryotic vegetative mycelium. However, it is a phenomenon which is not necessary for the completion of the life cycle. Whereas diploidy represents a somewhat exceptional nuclear phase in the vegetative mycelium of other higher basidiomycetes, in Armillariella diploidy seems to be the dominant nuclear phase and heterokaryosis a transient phase. The peculiar life cycle of Armillariella, although not yet completely solved, raises interesting questions about its origin and effect on evolution in this group of fungi with high variability.

Acknowledgements. I thank Dr. Henri Romagnesi, Dr. Micheline Jacques-Félix, and other members of the Société Mycologique de France for their indispensable help in collecting and determining the material from France. I am grateful to many people in Finland for the Finnish Armillariella material and to Mrs. Helga Marxmüller, Dr. John Rishbeth, Dr. Finn Roll-Hansen, Dr. Kazimierz Rykowski, and Dr. Charles G. Shaw III for material from other countries. Dr. Robert C. Ullrich and Mr. James B. Anderson have kindly provided me with a manuscript of their yet unpublished article. Dr. Tuomo Niemelä has made valuable suggestions concerning the manuscript. Especially I would like to thank Dr. Veikko Hintikka for the initiation fo this study, for critical appraisal of the manuscript, and for his keen interest.

\section{References}

Adams, D.H. 1974: Identification of clones of Armillaria mellea in young-growth Ponderosa pine. - Northwest Science 48: $21-28$.

Adams, D.H. \& Roth, L.F. 1967: Demarcation lines in paired cultures of Fomes cajanderi as a basis for detecting genetically distinct mycelia. - Canad. J. Bot. 45: $1583-1589$.

Bruehl, G.W., Machtmes, R. \& Kiyomoto, R. 1975: Taxonomic relationships among Typhula species as revealed by mating experiments. - Phytopathology 65 : $1108-1114$.

Edwards, R. \& Kennedy, L.L. 1973: Biosystematics of Coriolus hirsutus and C. pubescens 1. Interfertility studies. - Canad. J. Bot. 51: 2385-2393.

Fries, E.M. 1821: Systema mycologicum 1. - 520 pp. Lundae.

Hintikka, V. 1973: A note on polarity of Armillariella mellea. - Karstenia 13: 32-39.

- " - 1974: Notes on the ecology of Armillariella mellea in Finland. - Karstenia 14: 12-31.

Jacques-Félix, M. 1977: Le complexe de l'Armillaire. - Trav. Déd. G. Viennot-Bourgin 1977: 143-157.

Kallio, T. 1970: Aerial distribution of the root-rot fungus Fomes annosus (Fr.) Cooke in Finland. - Acta Forest. Fenn. 107: 1-55.

Kallio, T. \& Tamminen, P. 1974: Decay of spruce (Picea abies (L.) Karst.) in the Alland Islands. - Acta For. Fenn. 138: 1-44.

Karsten, P.A. 1881: Hymenomycetes fennici. - Acta Soc. Fauna Flora Fenn. 2: 1-40.

Kniep, H. 1911: Über das Auftreten von Basidien im einkernigen Mycel von Armillaria mellea Fl. Dan. - Z Bot. 3: $529-553$.

Koltin, Y. \& Raper, J.R. 1968: Dikaryosis: Genetic determination in Schizophyllum. - Science 160: $85-86$.

Korhonen, K. \& Hintikka, V. 1974: Cytological evidence for somatic diploidization in dikaryotic cells of Armillariella mellea. - Arch. Microbiol. 95: 187-192.

Kummer, P. 1871: Der Führer in die Pilzkunde. - 146 pp. Zerbst.

Martin, K.J. \& Gilbertson, R.L. 1978: Decay of Douglasfir by Sparassis radicata in Arizona. - Phytopathology 68: $149-154$.

Miles, P.G. \& Raper, J.R. 1956: Recovery of the component strains from dikaryotic mycelia. Mycologia 48: 484-494.

Osborne, L.D. 1970: Use of chemical monokaryotization in experimental taxonomy of Trametes lilacino-gilva and related species. - Trans. Brit. Mycol. Soc. 54: $283-287$.

Peabody, D.C., Motta, J.J. \& Therrien, C.D. 1978: Cytophotometric evidence for heteroploidy in the life cycle of Armillaria mellea. - Mycologia 70: 487-498.

Quélet, L. 1872: Les champignons du Jura et des Vosges (1). - Mém. Soc. Émul. Montbéliard (II) 5: 42-332.

Raper, J.R. 1966: Genetics of sexuality in higher fungi. -273 pp. New York.

Rishbeth, J. 1968: The growth rate of Armillaria mellea. - Trans. Brit. Mycol. Soc. 51: 576-586.

" - 1970: The role of basidiospores in stump infection by 
Armillaria mellea. - In: Tousson, T.A., Bega, R.V., Nelson, P.E. (eds.): Root diseases and soil-borne plant pathogens, pp. 141-146. Berkeley, Los Angeles, London.

Romagnesi, H. 1970: Observations sur les Armillariella (1). - Bull. Soc. Mycol. France 86: 257-268.

- " - 1973: Observations sur les Armillariella (2). - Bull. Soc. Mycol. France 89: 195-206.

Ross, I.K. 1976: Nuclear migration rates in Coprinus congregatus: a new record. - Mycologia 68: 418-422.

Rykowski, K. 1974: Observations on fructification of the honey fungus Armillariella mellea (Vahl) Karst. in pure cultures (in Polish, Engl. summ.). - Prace Inst. Badawczego Leśnictwa 431: 1-21.

Shaw, C.G., III \& Roth, L.F., 1976: Persistence and distribution of a clone of Armillaria mellea in a ponderosa pine forest. - Phytopathology 66: $1210-1213$.

Singer, R. 1956: The Armillariella mellea group. - Lloydia 19: $176-187$.

- " - 1975: The Agaricales in modern taxonomy. 3rd ed. 912 pp. Vaduz.

- " - 1977: An early fruiting Armillariella. - Kew Bull. 31: $433-435$

Tommerup, I.C. \& Broadbent, D. 1975: Nuclear fusion, meiosis and the origin of dikaryotic hyphae in Armillariella mellea. - Arch. Microbiol. 103: 279-282.

Ullrich, R.C. \& Anderson, J.B. 1978: Sex and diploidy in Armillaria mellea. - Experimental Mycology, in press.

Vahl, M. 1790: Flora Danica. Vol. 6. Fasc. 17, pl. 1013. Copenhagen.

Accepted for publication

on October 11, 1978 